MATHEMATICS OF COMPUTATION

Volume 68, Number 226, April 1999, Pages 651-659

S 0025-5718(99)01056-X

\title{
A QUASI-RANDOMIZED RUNGE-KUTTA METHOD
}

\author{
IBRAHIM COULIBALY AND CHRISTIAN LÉCOT
}

\begin{abstract}
We analyze a quasi-Monte Carlo method to solve the initial-value problem for a system of differential equations $y^{\prime}(t)=f(t, y(t))$. The function $f$ is smooth in $y$ and we suppose that $f$ and $D_{y}^{1} f$ are of bounded variation in $t$ and that $D_{y}^{2} f$ is bounded in a neighborhood of the graph of the solution. The method is akin to the second order Heun method of the Runge-Kutta family. It uses a quasi-Monte Carlo estimate of integrals. The error bound involves the square of the step size as well as the discrepancy of the point set used for quasi-Monte Carlo approximation. Numerical experiments show that the quasi-randomized method outperforms a recently proposed randomized numerical method.
\end{abstract}

\section{INTRODUCTION}

The Monte Carlo method is a very general tool for solving various problems of mathematical physics, and its applications are not restricted to numerical integration. It may be described as a numerical method based on random sampling. A good deal of effort has recently been directed to the use of quasi-Monte Carlo methods. A quasi-Monte Carlo method can be described as the deterministic variant of a Monte Carlo method, in the sense that the random samples are replaced by judiciously chosen deterministic points. For instance, in the area of numerical integration it is irrelevant whether the sample points are random. Of primary importance is the even distribution of the points. A review of the development of this area is given in the monograph [3] by Niederreiter.

A randomized algorithm for solving the initial value problem for the finite dimensional system

$$
\begin{aligned}
y^{\prime}(t) & =f(t, y(t)), \quad 0<t<T, \\
y(0) & =y_{0}
\end{aligned}
$$

was recently proposed by Stengle in [5]. The hypothesis is that $f$ is smooth in space $(y)$ but no more than bounded and measurable in time $(t)$. The algorithm is a member of a family akin to the Runge-Kutta family. It generates a sequence $Y_{n}$ by the recurrence formula

$$
\begin{gathered}
Y_{n+1}=Y_{n}+\frac{h_{n}}{2 N} \sum_{0 \leq j<N}\left(f\left(U_{j, n}, Y_{n}+h_{n} f\left(u_{j, n}, Y_{n}\right)\right)+f\left(u_{j, n}, Y_{n}\right)\right), \\
U_{j, n}=\max \left(T_{1, j, n}, T_{2, j, n}\right), \quad u_{j, n}=\min \left(T_{1, j, n}, T_{2, j, n}\right),
\end{gathered}
$$

Received by the editor July 18, 1997.

1991 Mathematics Subject Classification. Primary 65L06; Secondary 65C05.

Key words and phrases. Runge-Kutta method, quasi-Monte Carlo method, discrepancy.

(C)1999 American Mathematical Society 
where for each step $\left\{T_{1, j, n}: 0 \leq j<N\right\}$ and $\left\{T_{2, j, n}: 0 \leq j<N\right\}$ are fresh $N$-fold random samples of the uniform distribution on $\left[t_{n}, t_{n+1}\right]$. The entire family is called the Runge-Kutta Monte Carlo (RKMC) family. Stengle indicates that these methods can enjoy some advantages compared to standard methods under two conditions. First, the rate at which $f$ varies in $y$ must be significantly smaller than the rate at which $f$ varies in $t$ (a complete lack of regularity in $t$ being an extreme case). Second, the speedup which can be gained by parallel computation of the Monte Carlo estimates must be of consequence.

It may be hoped that the improvement achieved by using evenly distributed points in place of random numbers in integration problems can also be attained in initial-value problems. In this paper we propose a Runge-Kutta quasi-Monte Carlo (RKQMC) method which is of second order. It makes use of point sets with small discrepancy. Let $\lambda_{s}$ be the $s$-dimensional Lebesgue measure. For a point set $X$ consisting of $N$ points $\mathbf{x}_{0}, \ldots, \mathbf{x}_{N-1}$ in the half-open unit cube $I^{s}=[0,1)^{s}$ and a measurable subset $E$ of $I^{s}$, the local discrepancy is defined by

$$
D_{N}(E, X)=\frac{A(E, X)}{N}-\lambda_{s}(E),
$$

where $A(E, X)$ is the number of $j, 0 \leq j<N$, with $\mathbf{x}_{j} \in E$. The discrepancy of the point set $X$ is given by

$$
D_{N}(X)=\sup _{J}\left|D_{N}(J, X)\right|,
$$

the supremum being taken over all half open subintervals of $I^{s}$. We refer to [2] for background material on this topic. The RKQMC method uses the same twodimensional low discrepancy point set $X$ for the quasi-Monte Carlo approximation at each time step.

The paper is organized as follows. In $\S 2$ we describe the RKQMC method. In $\S 3$ we derive an error bound. If $\eta$ is the maximum of the step size, the error is bounded by a linear combination of $\eta^{2}$ and $D_{N}(X)$. In $\S 4$ we consider the simple model problem proposed by Stengle. The numerical results indicate that a significant improvement in magnitude of error is achieved over the RKMC scheme. In $\S 5$ we summarize the main points and conclusions of the paper.

\section{A Runge-Kutta quasi-Monte Carlo method}

We consider the initial value problem for the $p$-dimensional system (1),(2) where $y_{0}$ is given in $\mathbf{R}^{p}$. We assume that the problem is uniquely solvable and that the solution $y(t)$ is absolutely continuous on $[0, T]$. Let $\|\cdot\|$ be a norm on $\mathbf{R}^{p}$ and let $B(y, \rho)$ denote the corresponding open ball with center at $y$ and radius $\rho$. We assume that $f$ satisfies the following hypothesis.

Hypothesis. There exist $\tau>0$ and $\rho>0$ such that

- For every $t \in[0, T]$ the function $y \rightarrow D_{y}^{m} f(t, y)$ is continuous on the open ball $B(y(t), \rho)$, for $0 \leq m \leq 2$.

- Let

$$
\Omega=\bigcup_{0 \leq t \leq T}[t, \min (t+\tau, T)] \times B(y(t), \rho) .
$$


For every $t \in[0, T]$ and every $y \in B(y(t), \rho)$,

1. the function $u \rightarrow D_{y}^{m} f(u, y)$ is defined on $[t, \min (t+\tau, T)]$ and is bounded by $\left\|D_{y}^{m} f\right\|_{\infty, \Omega}$, for $0 \leq m \leq 2$; and

2. the variation of the function $u \rightarrow D_{y}^{m} f(u, y)$ on $[t, \min (t+\tau, T)]$ is bounded by $V_{\Omega}\left(D_{y}^{m} f\right)$, for $0 \leq m \leq 1$.

The following notation will be used: if $\mathbf{v}$ is a vector with coordinates $\left(v_{1}, v_{2}\right)$, we write $\underline{v}=\min \left(v_{1}, v_{2}\right)$ and $\bar{v}=\max \left(v_{1}, v_{2}\right)$. We consider a partition $0=t_{0}<$ $t_{1}<\ldots<t_{\nu}=T$ of $[0, T]$ into $\nu$ subintervals of length $h_{n}=t_{n+1}-t_{n}$ and we set $\eta=\max _{0 \leq n<\nu} h_{n}$. We have

$$
y\left(t_{n+1}\right)=y\left(t_{n}\right)+\int_{t_{n}}^{t_{n+1}} f\left(t, y\left(t_{n}\right)+\int_{t_{n}}^{t} f(u, y(u)) d u\right) d t .
$$

Through Taylor expansion in $y$ one has approximately

$$
\begin{aligned}
y\left(t_{n+1}\right) \approx & y\left(t_{n}\right) \\
& +\frac{1}{2 h_{n}} \int_{t_{n}}^{t_{n+1}} \int_{t_{n}}^{t_{n+1}}\left(f\left(\underline{s}, y\left(t_{n}\right)\right)+f\left(\bar{s}, y\left(t_{n}\right)+h_{n} f\left(\underline{s}, y\left(t_{n}\right)\right)\right)\right) d \mathbf{s} .
\end{aligned}
$$

The method of Heun [6] corresponds to the approximation $\underline{s} \approx t_{n}, \bar{s} \approx t_{n+1}$. Let $X$ be a point set consisting of $\mathbf{x}_{0}, \mathbf{x}_{1}, \ldots, \mathbf{x}_{N-1} \in \bar{I}^{2}$. The second order RKQMC method generates a sequence $\left(y_{n}\right)$ by

$$
\begin{aligned}
y_{n+1}= & y_{n} \\
& +\frac{h_{n}}{2 N} \sum_{0 \leq j<N}\left(f\left(t_{n}+h_{n} \underline{x}_{j}, y_{n}\right)+f\left(t_{n}+h_{n} \bar{x}_{j}, y_{n}+h_{n} f\left(t_{n}+h_{n} \underline{x}_{j}, y_{n}\right)\right)\right) .
\end{aligned}
$$

\section{BOUNDS FOR THE ERRORS}

The error analysis follows the general outline of the error analysis of Heun's scheme. The local discretization error is defined by

$$
\begin{aligned}
\varepsilon_{n}= & \frac{1}{h_{n}}\left(y\left(t_{n+1}\right)-y\left(t_{n}\right)\right) \\
& -\frac{1}{2 h_{n}^{2}} \int_{t_{n}}^{t_{n+1}} \int_{t_{n}}^{t_{n+1}}\left(f\left(\underline{s}, y\left(t_{n}\right)\right)+f\left(\bar{s}, y\left(t_{n}\right)+h_{n} f\left(\underline{s}, y\left(t_{n}\right)\right)\right)\right) d \mathbf{s} .
\end{aligned}
$$

We introduce an error term

$$
\begin{aligned}
E_{n}= & \frac{1}{2 h_{n}^{2}} \int_{t_{n}}^{t_{n+1}} \int_{t_{n}}^{t_{n+1}}\left(f\left(\underline{s}, y_{n}\right)+f\left(\bar{s}, y_{n}+h_{n} f\left(\underline{s}, y_{n}\right)\right)\right) d \mathbf{s} \\
& -\frac{1}{2 h_{n}^{2}} \int_{t_{n}}^{t_{n+1}} \int_{t_{n}}^{t_{n+1}}\left(f\left(\underline{s}, y\left(t_{n}\right)\right)+f\left(\bar{s}, y\left(t_{n}\right)+h_{n} f\left(\underline{s}, y\left(t_{n}\right)\right)\right)\right) d \mathbf{s},
\end{aligned}
$$

and the error of the quasi-Monte Carlo approximation

$$
\begin{aligned}
\delta_{n}= & \frac{1}{2 N} \sum_{0 \leq j<N}\left(f\left(t_{n}+h_{n} \underline{x}_{j}, y_{n}\right)+f\left(t_{n}+h_{n} \bar{x}_{j}, y_{n}+h_{n} f\left(t_{n}+h_{n} \underline{x}_{j}, y_{n}\right)\right)\right) \\
& -\frac{1}{2} \int_{I^{2}}\left(f\left(t_{n}+h_{n} \underline{x}, y_{n}\right)+f\left(t_{n}+h_{n} \bar{x}, y_{n}+h_{n} f\left(t_{n}+h_{n} \underline{x}, y_{n}\right)\right)\right) d \mathbf{x} .
\end{aligned}
$$


The global discretization error is $e_{n}=y_{n}-y\left(t_{n}\right)$. We have the recurrence formula

$$
e_{n+1}=e_{n}-h_{n} \varepsilon_{n}+h_{n} E_{n}+h_{n} \delta_{n} .
$$

We need an appropriate concept of total variation for functions of several variables. For a function $\varphi: \bar{I}^{s} \rightarrow \mathbf{R}^{p}$ and for $\mathbf{w}, \mathbf{w}^{\prime} \in \bar{I}^{s}$ and $1 \leq i \leq s$, let $T_{\mathbf{w}}^{i} \varphi$ be the restriction of $\varphi$ to the hyperplane $x_{i}=w_{i}$ and $\Delta_{\mathbf{w}, \mathbf{w}^{\prime}}^{i} \varphi=T_{\mathbf{w}^{\prime}}^{i} \varphi-T_{\mathbf{w}}^{i} \varphi$. If $K=\left\{i_{1}, \ldots, i_{k}\right\} \subset[1, s]$, we set

$$
T_{\mathbf{w}}^{K} \varphi=T_{\mathbf{w}}^{i_{1}} \cdots T_{\mathbf{w}}^{i_{k}} \varphi \quad \text { and } \quad \Delta_{\mathbf{w}, \mathbf{w}^{\prime}}^{K} \varphi=\Delta_{\mathbf{w}, \mathbf{w}^{\prime}}^{i_{1}} \cdots \Delta_{\mathbf{w}, \mathbf{w}^{\prime}}^{i_{k}} \varphi .
$$

We put $T_{\mathbf{w}} \varphi=T_{\mathbf{w}}^{[1, s]} \varphi$ and $\Delta_{\mathbf{w}, \mathbf{w}^{\prime}} \varphi=\Delta_{\mathbf{w}, \mathbf{w}^{\prime}}^{[1, s} \varphi$. If

$$
0=x_{0, i}<x_{1, i}<\cdots<x_{n_{i}, i}=1, \quad \text { for } \quad 1 \leq i \leq s
$$

define a partition of $\bar{I}^{s}$ into subintervals, and $\mathbf{a}=\left(a_{1}, \ldots, a_{s}\right)$ with integers $a_{i}, 0 \leq$ $a_{i}<n_{i}$, we write $\mathbf{x}_{\mathbf{a}}=\left(x_{a_{1}, 1}, \ldots, x_{a_{s}, s}\right)$ and $\mathbf{a}+=\left(a_{1}+1, \ldots, a_{s}+1\right)$. The variation of $\varphi$ on $\bar{I}^{s}$ in the sense of Vitali is defined by

$$
V^{(s)}(\varphi)=\sup _{\mathcal{P}} \sum_{\mathbf{a}}\left\|\Delta_{\mathbf{x}_{\mathbf{a}}, \mathbf{x}_{\mathbf{a}+}} \varphi\right\|
$$

where $\mathcal{P}$ runs through all partitions of $\bar{I}^{s}$ into subintervals. Let $\mathbf{1}=(1, \ldots, 1) \in \bar{I}^{s}$, then

$$
V(\varphi)=\sum_{k=1}^{s} \sum_{\substack{K \subset[1, s] \\ \# K=k}} V^{(k)}\left(T_{1}^{K^{c}} \varphi\right)
$$

is called the variation of $\varphi$ on $\bar{I}^{s}$ in the sense of Hardy and Krause. We refer to [2] for further information on this concept. The class of all functions of bounded variation on $\bar{I}^{s}$ in the sense of Hardy and Krause will be denoted by $B V H K^{s}$. By [7, Proposition 2], every function in $B V H K^{s}$ is Riemann-integrable on $\bar{I}^{s}$. If $\varphi$ is a function defined on $\bar{I}^{2}$, we write $\varphi^{o}(\mathbf{x})=\varphi(\underline{x}, \bar{x})$. We need the following technical results.

Lemma 1. If $\varphi \in B V H K^{2}$, then $\varphi^{o} \in B V H K^{2}$.

Proof. We have $T_{1}^{1} \varphi^{o}=T_{1}^{2} \varphi^{o}=T_{1}^{2} \varphi$. A short calculation shows that

$$
V^{(2)}\left(\varphi^{o}\right) \leq 3 V^{(2)}(\varphi)+V^{(1)}\left(T_{1}^{1} \varphi\right)+V^{(1)}\left(T_{1}^{2} \varphi\right),
$$

and the result of the lemma follows.

If $t \in[0, T], 0 \leq h$ and $y \in \mathbf{R}^{p}$, define

$$
\begin{aligned}
\phi_{t, h, y}(\mathbf{x}) & =f\left(t+h x_{1}, y\right), \\
\psi_{t, h, y}(\mathbf{x}) & =f\left(t+h x_{2}, y+h f\left(t+h x_{1}, y\right)\right),
\end{aligned}
$$

for $\mathbf{x} \in \bar{I}^{2}$.

Lemma 2. If $t$ and $t+h \in[0, T], h \leq \tau$ and $\|y-y(t)\|+2 h\|f\|_{\infty, \Omega}<\rho$, then $\phi_{t, h, y}$ and $\psi_{t, h, y} \in B V H K^{2}$. 
Proof. Straightforward calculations show that

$$
\begin{aligned}
& V^{(1)}\left(T_{\mathbf{1}}^{1} \phi_{t, h, y}\right)=V^{(2)}\left(\phi_{t, h, y}\right)=0, \quad V^{(1)}\left(T_{\mathbf{1}}^{2} \phi_{t, h, y}\right) \leq V_{\Omega}(f), \\
& V^{(1)}\left(T_{\mathbf{1}}^{1} \psi_{t, h, y}\right) \leq V_{\Omega}(f) .
\end{aligned}
$$

Through Taylor expansion in $y$ one finds

$$
V^{(1)}\left(T_{1}^{2} \psi_{t, h, y}\right) \leq h V_{\Omega}(f)\left\|D_{y}^{1} f\right\|_{\infty, \Omega} \quad \text { and } \quad V^{(2)}\left(\psi_{t, h, y}\right) \leq h V_{\Omega}(f) V_{\Omega}\left(D_{y}^{1} f\right) .
$$

This completes the proof.

We shall put

$$
\phi_{n}=\phi_{t_{n}, h_{n}, y_{n}}, \quad \Phi_{n}=\phi_{t_{n}, h_{n}, y\left(t_{n}\right)}, \quad \psi_{n}=\psi_{t_{n}, h_{n}, y_{n}}, \quad \Psi_{n}=\psi_{t_{n}, h_{n}, y\left(t_{n}\right)} .
$$

Then

$$
\begin{aligned}
\varepsilon_{n} & =\frac{1}{h_{n}}\left(y\left(t_{n+1}\right)-y\left(t_{n}\right)\right)-\frac{1}{2} \int_{I^{2}}\left(\Phi_{n}^{o}(\mathbf{x})+\Psi_{n}^{o}(\mathbf{x})\right) d \mathbf{x}, \\
E_{n} & =\frac{1}{2} \int_{I^{2}}\left(\phi_{n}^{o}(\mathbf{x})+\psi_{n}^{o}(\mathbf{x})\right) d \mathbf{x}-\frac{1}{2} \int_{I^{2}}\left(\Phi_{n}^{o}(\mathbf{x})+\Psi_{n}^{o}(\mathbf{x})\right) d \mathbf{x}, \\
\delta_{n} & =\frac{1}{2 N} \sum_{0 \leq j<N}\left(\phi_{n}^{o}\left(\mathbf{x}_{j}\right)+\psi_{n}^{o}\left(\mathbf{x}_{j}\right)\right)-\frac{1}{2} \int_{I^{2}}\left(\phi_{n}^{o}(\mathbf{x})+\psi_{n}^{o}(\mathbf{x})\right) d \mathbf{x} .
\end{aligned}
$$

We now establish upper bounds for these terms, for $0 \leq n<\nu$.

Proposition 1. If $h_{n} \leq \tau$ and $2 h_{n}\|f\|_{\infty, \Omega}<\rho$, then

$$
\left\|\varepsilon_{n}\right\| \leq \frac{h_{n}^{2}}{12}\|f\|_{\infty, \Omega}\left(5\|f\|_{\infty, \Omega}\left\|D_{y}^{2} f\right\|_{\infty, \Omega}+2\left\|D_{y}^{1} f\right\|_{\infty, \Omega}^{2}\right) .
$$

Proof. By Lemmas 1 and $2, \varepsilon_{n}$ is well defined. By using Taylor expansions in $y$ we obtain $\varepsilon_{n}=-\varepsilon_{n, 1}+\varepsilon_{n, 2}+\varepsilon_{n, 3}$, where

$$
\begin{aligned}
& \varepsilon_{n, 1}= \frac{h_{n}^{2}}{2} \int_{I^{2}}\left(\int_{I} D_{y}^{2} f\left(t_{n}+h_{n} \bar{x}, y\left(t_{n}\right)+\xi h_{n} f\left(t_{n}+h_{n} \underline{x}, y\left(t_{n}\right)\right)\right)\right. \\
& \varepsilon_{n, 2}= \frac{h_{n}}{2} \int_{I^{2}} D_{y}^{1} f\left(t_{n}+h_{n} \bar{x}, y\left(t_{n}\right)\right) \\
&\left.\left.\cdot\left(\int_{I} D_{y}^{1} f\left(t_{n}+h_{n} \underline{x}, y\left(t_{n}\right)\right)\right)^{2}(1-\xi) d \xi\right) d \mathbf{x},(1-\xi) y\left(t_{n}\right)+\xi y\left(t_{n}+h_{n} \underline{x}\right)\right) \\
& \varepsilon_{n, 3}=\left.\int_{I}\left(\int_{I} D_{y}^{2} f\left(t_{n}+h_{n} x,(1-\xi) y\left(t_{n}\right)+\xi y(t)\right)-y\left(t_{n}\right)\right) d \xi\right) d \mathbf{x}, \\
&\left.\cdot\left(y\left(t_{n}+h_{n} x\right)-y\left(t_{n}\right)\right)^{2}(1-\xi) d \xi\right) d x .
\end{aligned}
$$

Hence the result of the proposition follows.

Proposition 2. If $h_{n} \leq \tau$ and $\left\|e_{n}\right\|+2 h_{n}\|f\|_{\infty, \Omega}<\rho$, then

$$
\left\|E_{n}\right\| \leq\left\|D_{y}^{1} f\right\|_{\infty, \Omega}\left(1+\frac{h_{n}}{2}\left\|D_{y}^{1} f\right\|_{\infty, \Omega}\right)\left\|e_{n}\right\| .
$$


Proof. We know from Lemmas 1 and 2 that each integral in (7) exists. By Taylor expansions in $y$, we find $E_{n}=E_{n, 1}+E_{n, 2}$, where

$$
\begin{array}{r}
E_{n, 1}=\frac{1}{2} \int_{I^{2}}\left(\int_{I} D_{y}^{1} f\left(t_{n}+h_{n} \underline{x},(1-\xi) y\left(t_{n}\right)+\xi y_{n}\right) \cdot\left(y_{n}-y\left(t_{n}\right)\right) d \xi\right) d x \\
E_{n, 2}=\frac{1}{2} \int_{I^{2}}\left(\int _ { I } D _ { y } ^ { 1 } f \left(t_{n}+h_{n} \bar{x},(1-\xi)\left(y\left(t_{n}\right)+h_{n} f\left(t_{n}+h_{n} \underline{x}, y\left(t_{n}\right)\right)\right)\right.\right. \\
\left.+\xi\left(y_{n}+h_{n} f\left(t_{n}+h_{n} \underline{x}, y_{n}\right)\right)\right) \\
\left.\cdot\left(y_{n}+h_{n} f\left(t_{n}+h_{n} \underline{x}, y_{n}\right)-y\left(t_{n}\right)-h_{n} f\left(t_{n}+h_{n} \underline{x}, y\left(t_{n}\right)\right)\right) d \xi\right) d \mathbf{x}
\end{array}
$$

so that the desired bound for $\left\|E_{n}\right\|$ follows.

A bound for $\delta_{n}$ is established by using techniques of Zaremba ([7]).

Proposition 3. If $h_{n} \leq \tau$ and $\left\|e_{n}\right\|+2 h_{n}\|f\|_{\infty, \Omega}<\rho$, then

$$
\left\|\delta_{n}\right\| \leq V_{\Omega}(f)\left(1+\frac{h_{n}}{2}\left(\left\|D_{y}^{1} f\right\|_{\infty, \Omega}+2 V_{\Omega}\left(D_{y}^{1} f\right)\right)\right) D_{N}(X) .
$$

Proof. We consider a double partition of $\bar{I}$ :

$$
\begin{gathered}
0=w_{0}<w_{1}<\cdots<w_{\ell}=1, \\
\xi_{0}=0, \quad w_{k} \leq \xi_{k+1}<w_{k+1}, \text { for } 0 \leq k<\ell, \quad \xi_{\ell+1}=1,
\end{gathered}
$$

such that $X \subset\left\{\xi_{\mathbf{k}}=\left(\xi_{k_{1}}, \xi_{k_{2}}\right): 0 \leq k_{1}, k_{2} \leq \ell+1\right\}$. Put

$$
h(W)=\max _{0 \leq k<\ell}\left(w_{k+1}-w_{k}\right)
$$

By using the multidimensional Abel summation formula with the auxiliary function

$$
\zeta(\mathbf{x})=D_{N}\left(\left[0, x_{1}\right) \times\left[0, x_{2}\right), X\right),
$$

one finds for sufficiently small $h(W)$,

$$
\begin{aligned}
\frac{1}{N} \sum_{0 \leq j<N} \phi_{n}^{o}\left(\mathbf{x}_{j}\right)-\sum_{0 \leq k_{1}, k_{2}<\ell}\left(w_{k_{1}+1}-w_{k_{1}}\right)\left(w_{k_{2}+1}-w_{k_{2}}\right) \phi_{n}^{o}\left(\xi_{k_{1}+1}, \xi_{k_{2}+1}\right) \\
=\sum_{0 \leq k \leq \ell}\left(f\left(t_{n}+h_{n} \xi_{k+1}, y_{n}\right)-f\left(t_{n}+h_{n} \xi_{k}, y_{n}\right)\right) D_{N}\left(\left[w_{k}, 1\right)^{2}, X\right)
\end{aligned}
$$


A QUASI-RANDOMIZED RUNGE-KUTTA METHOD

and

$$
\begin{aligned}
\frac{1}{N} \sum_{0 \leq j<N} \psi_{n}^{o}\left(\mathbf{x}_{j}\right)- & \sum_{0 \leq k_{1}, k_{2}<\ell}\left(w_{k_{1}+1}-w_{k_{1}}\right)\left(w_{k_{2}+1}-w_{k_{2}}\right) \psi_{n}^{o}\left(\xi_{k_{1}+1}, \xi_{k_{2}+1}\right) \\
= & -\sum_{0 \leq k_{1}<k_{2} \leq \ell} \Delta_{\xi_{\mathbf{k}}, \xi_{\mathbf{k}+}} \psi_{n} D_{N}\left(\left[w_{k_{1}}, w_{k_{2}}\right)^{2}, X\right) \\
& +\sum_{0 \leq k_{1}, k_{2} \leq \ell} \Delta_{\xi_{\mathbf{k}}, \xi_{\mathbf{k}+}} \psi_{n} D_{N}\left(\left[0, w_{k_{2}}\right)^{2}, X\right) \\
& -\sum_{0 \leq k \leq \ell} \Delta_{\xi_{k}, \xi_{k+}} T_{\mathbf{1}}^{1} \psi_{n} D_{N}\left(\left[0, w_{k}\right)^{2}, X\right) \\
& +\sum_{0 \leq k \leq \ell} \Delta_{\xi_{k}, \xi_{k+}} T_{\mathbf{1}}^{2} \psi_{n} D_{N}\left(\left[w_{k}, 1\right)^{2}, X\right) .
\end{aligned}
$$

Passing to the limit $h(W) \rightarrow 0$ and using Lemma 1 and the estimates of Lemma 2, we obtain the desired bound.

We are now in a position to prove the convergence result. Put

$$
\begin{aligned}
c_{1}(f) & =\frac{1}{12}\|f\|_{\infty, \Omega}\left(5\|f\|_{\infty, \Omega}\left\|D_{y}^{2} f\right\|_{\infty, \Omega}+2\left\|D_{y}^{1} f\right\|_{\infty, \Omega}^{2}\right), \\
c_{2}(f, \eta) & =\left\|D_{y}^{1} f\right\|_{\infty, \Omega}\left(1+\frac{\eta}{2}\left\|D_{y}^{1} f\right\|_{\infty, \Omega}\right), \\
c_{3}(f, \eta) & =V_{\Omega}(f)\left(1+\frac{\eta}{2}\left(\left\|D_{y}^{1} f\right\|_{\infty, \Omega}+2 V_{\Omega}\left(D_{y}^{1} f\right)\right)\right) .
\end{aligned}
$$

Proposition 4. If $\eta \leq \tau$ and

$$
e^{c_{2}(f, \eta) T}\left\|e_{0}\right\|+\frac{e^{c_{2}(f, \eta) T}-1}{c_{2}(f, \eta)}\left(c_{1}(f) \eta^{2}+c_{3}(f, \eta) D_{N}(X)\right)+2 \eta\|f\|_{\infty, \Omega}<\rho,
$$

then, for $0 \leq n \leq \nu$,

$$
\left\|e_{n}\right\| \leq e^{c_{2}(f, \eta) t_{n}}\left\|e_{0}\right\|+\frac{e^{c_{2}(f, \eta) t_{n}}-1}{c_{2}(f, \eta)}\left(c_{1}(f) \eta^{2}+c_{3}(f, \eta) D_{N}(X)\right) .
$$

Proof. Use (5) and combine it with Propositions 1, 2 and 3.

By a classical result (see [4, Theorem 2]), the discrepancy of any $N$-element point set $X$ in dimension two satisfies

$$
D_{N}(X) \geq c \frac{\log N}{N}
$$

where $c>0$ is an absolute constant. In [3], Niederreiter provides a survey of numerous low-discrepancy point sets. For integers $N \geq 1$ and $b \geq 2$, the $N$-element Hammersley point set in base $b$ is given by

$$
X=\left\{\left(\varphi_{b}(j), j / N\right): 0 \leq j<N\right\},
$$

where $\varphi_{b}$ is the radical-inverse function in base $b$. Then by [3, Theorem 3.8]

$$
D_{N}(X)<4\left(\frac{2}{N}+\frac{1}{N}\left(\frac{b-1}{2 \log b} \log N+\frac{b+1}{2}\right)\right) .
$$

So the discrepancy of an $N$-element Hammersley point set attains the least possible order of magnitude.

According to the estimate of Proposition 4, the RKQMC algorithm using an $\mathrm{N}$ element Hammersley point set is of second order if $N=O\left(\eta^{-2}\right)$. Stengle states that 
the RKMC method yields the following error bound. There is a positive constant $c$ such that, given a level $\beta>0$, then with probability not less than $1-\beta$

$$
\left\|e_{n}\right\| \leq c\left(\eta^{2}+\left(\frac{\eta}{\beta N}\right)^{1 / 2} S\left(f, t_{n}, \eta\right)\right),
$$

where $S$ is bounded and $S=O(\eta)$ if $f$ is smooth. According to this estimate, the RKMC algorithm is second order if $N=O\left(\eta^{-3}\right)$.

\section{A NUMERICAL EXAMPLE}

For illustration, we use second order methods to solve a simple model problem proposed by Stengle:

$$
\begin{aligned}
y^{\prime}(t) & =y(t)+\mu \sin (\cos (\lambda t)), \quad 0<t<1, \\
y(0) & =1,
\end{aligned}
$$

where $\lambda=1023$ and $\mu=5$. Figure 1 compares global discretization errors obtained in solving this problem using the following schemes.

- The method of Heun with 10 (RK10) and 100 (RK100) equally spaced steps.

- The RKMC method of Stengle with 10 equally spaced steps. It uses fresh 100-fold random samples of the uniform distribution for each step.

- The RKQMC method with 10 equally spaced time steps. It uses the 100element Hammersley point set in base 2.

The left panel shows RK10 (dashed line) and RKMC (solid line) results. The right panel shows RK100 (dashed line, only errors at time $t_{n}=n / 10,0 \leq n \leq 10$ are shown for clarity) and RKQMC (solid line) results. Note the difference in vertical scales. The parameters are selected so that the integration with the larger time step is at the limit of its capacity to follow the solution. The result of the RKMC algorithm with 10 time steps follows the finer RK integration quite closely. But the use of the Hammersley point set in quasi-Monte Carlo approximation leads to a dramatic improvement in the Monte Carlo error. Similar numerical experiments performed using several low-discrepancy point sets were equally promising for quasiMonte Carlo methods.

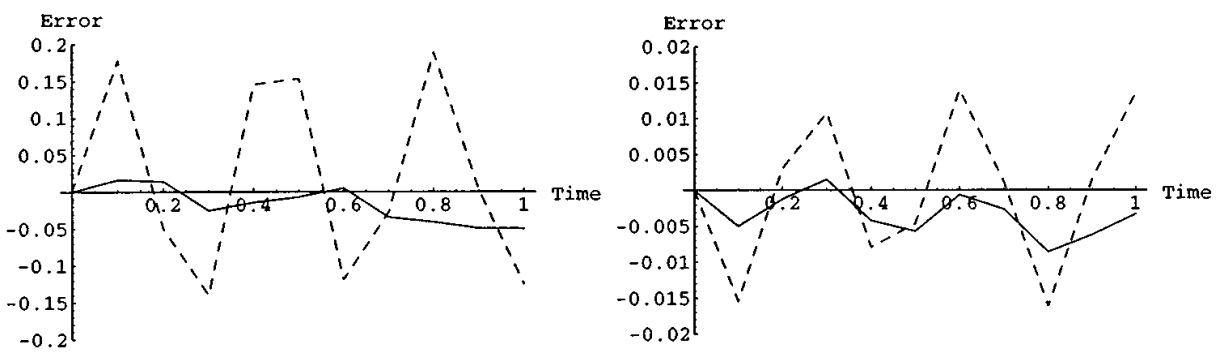

Figure 1. Comparison of second order Runge-Kutta methods. 


\section{Conclusion}

This paper demonstrates that a quasi-randomized Runge-Kutta method is feasible and can actually produce more accurate results than a randomized numerical method. It turns out that the randomness of the samples of the uniform distribution is irrelevant. The convergence analysis shows that if point sets with lower discrepancy than random sets are used, better than random convergence is possible. The numerical results confirm this. There are quasi-Monte Carlo methods for various other numerical problems. In fact, for many Monte Carlo methods it is possible to develop corresponding quasi-Monte Carlo methods as their deterministic versions. In a companion paper ([1]) we present a quasi-random walk method which outperforms standard random walk methods.

\section{REFERENCES}

1. I. Coulibaly and C. Lécot, Simulation of diffusion using quasi-random walk methods, Math. Comput. Simulation 47 (1998), 155-166.

2. L. Kuipers and H. Niederreiter, Uniform Distribution of Sequences, John Wiley \& Sons, New York, 1974. MR 87c:11066

3. H. Niederreiter, Random Number Generation and Quasi-Monte Carlo Methods, Society for Industrial and Applied Mathematics, Philadelphia, 1992. MR 93h:65008

4. W.M. Schmidt, Irregularities of distribution, VII, Acta Arith. 21 (1972), 45-50. MR 47:8474

5. G. Stengle, Error analysis of a randomized numerical method, Numer. Math. 70 (1995), 119128. MR 95m:65008

6. J. Stoer and R. Bulirsch, Introduction to Numerical Analysis, 2nd ed., Springer-Verlag, New York, 1993. MR 95i:65006

7. S.K. Zaremba, Some applications of multidimensional integration by parts, Ann. Polon. Math. 21 (1968), 85-96. MR 38:4034

Laboratoire de Mathématiques, Université de Savoie, Campus scientifique, 73376 Le Bourget-du-Lac CEDEx, France

E-mail address: Christian.Lecot@univ-savoie.fr 